\title{
Does Cisapride, as a 5HT 4 Receptor Agonist, Aggravate the Severity of TNBS-Induced Colitis in Rat?
}

\author{
Azadeh Motavallian, ${ }^{1}$ Mohsen Minaiyan, ${ }^{1,2}$ Mohammad Rabbani, ${ }^{1,2}$ \\ Parvin Mahzouni, ${ }^{3}$ Sasan Andalib, ${ }^{4}$ Alireza Abed, ${ }^{1}$ and Mohammad Reza Babavalian ${ }^{5}$ \\ ${ }^{1}$ Isfahan Pharmaceutical Sciences Research Center, School of Pharmacy and Pharmaceutical Sciences, \\ Isfahan University of Medical Sciences, Isfahan 8146-73461, Iran \\ ${ }^{2}$ Department of Pharmacology and Toxicology, Faculty of Pharmacy, Isfahan University of Medical Sciences, \\ Isfahan 8146-73461, Iran \\ ${ }^{3}$ Department of Clinical Pathology, School of Medicine, Isfahan University of Medical Sciences, Isfahan 8146-73461, Iran \\ ${ }^{4}$ Neurosciences Research Center, Tabriz University of Medical Sciences, Tabriz 8146-73461, Iran \\ ${ }^{5}$ Islamic Azad University, Qazvin Branch, Qazvin 8146-73461, Iran
}

Correspondence should be addressed to Mohsen Minaiyan, minaiyan@pharm.mui.ac.ir

Received 31 May 2012; Revised 9 June 2012; Accepted 9 June 2012

Academic Editor: A. Andoh

Copyright ( 2012 Azadeh Motavallian et al. This is an open access article distributed under the Creative Commons Attribution License, which permits unrestricted use, distribution, and reproduction in any medium, provided the original work is properly cited.

There is a pressing need for research that will lead to the reveal of targets designed to analyse the possible pathways for the treatment of IBD. Because of the probable involvement of serotonin in inflammatory conditions of intestine and the important role of $5 \mathrm{HT}_{4}$ receptors in GI function, the investigation of the role of $5 \mathrm{HT}_{4}$ receptors in the pathogenesis of IBD will be interesting. The aim of this study was to investigate the effects of cisapride, a $5 \mathrm{HT}_{4}$ receptor agonist, in trinitrobenzenesulfonic-acid-(TNBS) induced rat colitis. Two hours subsequent to induction of colitis using TNBS in rats, cisapride ( $2 \mathrm{mg} / \mathrm{kg}$, intraperitoneally (i.p); $4 \mathrm{mg} / \mathrm{kg}$, orally (p.o)) and dexamethasone ( $1 \mathrm{mg} / \mathrm{kg}$, i.p; $2 \mathrm{mg} / \mathrm{kg}, \mathrm{p} . \mathrm{o})$ were administrated for 6 days. Animals were thereafter euthanized; macroscopic, histological, and biochemical assessments and ELISA test were carried out on distal colon samples. Our data showed that dexamethasone treatment (i.p, p.o) significantly decreased macroscopic and microscopic damage and also biochemical markers, but there were no significant differences in aforementioned parameters between cisapride (i.p or p.o) and TNBS-treated rats. It can be deduced that because the severity of colitis produced by TNBS is massive (through various pathways), cisapride could not bring about more colitis damages through $5 \mathrm{HT}_{4}$ receptors. Based on the present study further researches are required for investigating the exact roles of $5 \mathrm{HT}_{4}$ receptors in the pathogenesis of ulcerative colitis.

\section{Introduction}

Inflammatory bowel disease (IBD), comprising ulcerative colitis (UC) and Crohn's disease (CD), is a chronic inflammatory disorder of the gastrointestinal (GI) tract characterized by a relapsing course [1]. Despite extensive research in the last decades, the etiology of IBD and the initial event of the inflammatory cascade still remain ambiguous [2]. In fact, genetic, immune, and environmental factors are involved in its pathogenesis $[2,3]$. IBD is characterized by massive cellular infiltrates and pertains to abnormalities of the immune system involving heightened number of $\mathrm{CD} 4^{+}$ $\mathrm{T}$ lymphocytes, mast cells, neutrophils, and eosinophils
[4]. These conditions bring about inflammation, ulceration, edema, diarrhea accompanied by blood and/or mucus, fever, abdominal pain and gastric dysmotility, anemia, weight loss, and a range of extraintestinal symptoms [5]. Many medical therapies have been proposed for IBD like salicylates, glucocorticoids, and immunosuppressives; nonetheless, available medicines are not universally effective and result in marked deleterious effects, and therefore the medical management of IBD remains challenging, and investigations on novel treatments are required [6].

5-hydroxytryptamine (5-HT, serotonin) is an important gastrointestinal (GI) signaling molecule involved in motor, 
secretory, and sensory functions. It is also found in the immune-inflammatory axis and affects the mammalian immune response $[7,8]$. These actions are mediated by a large family of serotonin receptors located within the neural circuitry and on a variety of other cell types in the gut [9]. Of the 5-HT receptors expressed in the intestines, the $5-\mathrm{HT}_{3}$ and $5 \mathrm{HT}_{4}$ receptors have been the most widely studied in regards to GI function [10]. Because of extensive distribution of $5 \mathrm{HT}_{3}$ and $5 \mathrm{HT}_{4}$ receptors in intestine and especially in colon, more recently interest is focusing on the roles of these two receptors as pharmacotherapeutic targets for the treatment of GI disorders [10]. In this regard $5-\mathrm{HT}_{3}$ receptor antagonists are used to treat nausea and emesis associated with chemotherapy [11] and for functional disorders associated with diarrhea [12]. In addition, recent investigations have revealed new clinical indications for this class of drugs. Preliminary data have shown the analgesic effects of ondansetron and tropisetron in patients with neuropathic pain and chronic inflammatory joint diseases, respectively $[13,14]$.

Animal models of IBD have been central to the investigation of the pathophysiology of the disease and are valuable tools for drug testing and development. The murine model of TNBS-induced acute or chronic colitis in rat is one of the most widely used models in both pharmacological and pathophysiological studies [15]. UC is said to be associated with the changes in EC cell numbers, 5-HT content, and enhancement of serotonin secretion. Additionally, an augmentation in the amount of 5-HT and secretion resembling the pattern of UC was seen in TNBS model of colitis [16].

In spite of the fact that the highest expression of $5 \mathrm{HT}_{4}$ receptors is in the distal colon [17], little is known about the role of this receptor in pathogenesis of GI diseases such as ulcerative colitis. Nowadays, $5-\mathrm{HT}_{4}$ receptor agonists are used as promotility agents to promote gastric emptying and to alleviate constipation. Cisapride, as a $5 \mathrm{HT}_{4}$ receptor agonist, was developed with the intention of treating both upper and lower gastrointestinal dysfunction (in particular, for gastroesophageal reflux disease and dyspepsia), but finally it has been removed from the United States market secondary to the risk of arrhythmias in those with predisposing conditions [10]. In addition, Hoffman et al. demonstrated the $5-\mathrm{HT}_{4}$ receptor expression by EC cells, goblet cells, and enterocytes [17]. Therefore the stimulation of serotonin secretion by any or all of these cell types through $5 \mathrm{HT}_{4}$ receptors could promote the cascades which 5HT is involved in them. Intestinal inflammation may arise from a change in 5-HT-producing enterochromaffin (EC) cells and an increase in 5-HT content associated with the pattern of IBD [18]. It is also worth noting that serotoninergic receptors were found in immune cells such as macrophages, a principle source of proinflammatory cytokines IL-1, IL-6, and TNF- $\alpha$ [19]. Although $5 \mathrm{HT}_{4}$ receptor mRNA was not detected in immune cells in animal studies [20], liberation of 5HT following activation of $5 \mathrm{HT}_{4}$ receptors could serve a critical role in infiltration and activation of macrophages via activation of their 5HT receptors, in intestinal inflammation [19, 21]. Due to the fact that increasing content of $5 \mathrm{HT}$ has demonstrated in UC, there may be an association between intestinal inflammation and a change in 5-HT content [18]. In addition in our previous study, we demonstrated that the blocking of $5 \mathrm{HT}_{3}$ receptors by ondansetron, a $5 \mathrm{HT}_{3}$ receptor antagonist, alleviated the colon injuries in experimental colitis [22]. Considering these data, the question arises as to whether the $5 \mathrm{HT}_{4}$ receptors like $5 \mathrm{HT}_{3}$ receptors are involved in pathogenesis of UC. If this hypothesis is true, it is likely that the activation of $5 \mathrm{HT}_{4}$ receptors deteriorates the severity of colitis. Therefore, our aim is to characterize the effect of administration of cisapride as a $5 \mathrm{HT}_{4}$ receptor agonist on an immune-based animal model of colitis (TNBS model) in rat.

\section{Methods}

2.1. Animals. Male Wistar rats $(200 \pm 20 \mathrm{~g})$ obtained from the laboratory animal house of School of Pharmacy, Isfahan University of Medical Sciences were randomly distributed into several experimental groups. Animal quarters maintained a constant temperature $\left(22 \pm 1^{\circ} \mathrm{C}\right)$, relative humidity $(55 \pm 10 \%)$, and a $12 \mathrm{~h}$ light-dark cycle. Rats fed standard pelleted chow and water ad libitum. Animals were treated in accordance with the Guide for the Care and Use of Laboratory Animals as adopted and promulgated by the Animal Care Committee of the Isfahan University of Medical Sciences.

2.2. Chemicals. Dexamethasone and cisapride were obtained from Iran Hormone Pharmaceutical Co. (Tehran, Iran) and Sigma Chemical Company (St. Louis, MO, USA), respectively. TNBS, hexadecyl trimethyl-ammonium bromide (HTAB), aprotinin A, bovine serum albumin, phenylmethylsulfonyl fluoride, benzethonium chloride, ethylene diamine tetra acetic acid (EDTA), and tween 20 were provided from Sigma Chemical Company (St. Louis, MO, USA). TNF- $\alpha$ (ALPCO, USA), IL-1 $\beta$ (ALPCO, USA), and IL-6 (ALPCO, USA) kits were used in order to analyze the biochemical variables.

2.3. Grouping. Rats were randomly assigned to 8 groups (6 animals in each) as follows. (Ia) TNBS-control group: rats received normal saline intraperitoneally (i.p) 2 hours following induction of colitis; (Ib) TNBS-control group: rats received normal saline orally (p.o) 2 hours following induction of colitis (IIa) normal group: cannulation was accomplished without induction of colitis (normal saline was administered instead of TNBS), and rats received normal saline i.p; (IIb) normal group: cannulation was accomplished without induction of colitis (normal saline was administered instead of TNBS), and rats received normal saline p.o; (IIIa) dexamethasone, i.p group: dexamethasone $(1 \mathrm{mg} / \mathrm{kg}$, i.p) was given 2 hours following induction of colitis [23]; (IIIb) dexamethasone, p.o group: dexamethasone $(2 \mathrm{mg} / \mathrm{kg}$, p.o) was given 2 hours following induction of colitis [23]; (IVa) cisapride, i.p group: cisapride $(2 \mathrm{mg} / \mathrm{kg}$, i.p) was administered 2 hours following induction of colitis [24]; (V) cisapride, p.o. group: cisapride ( $4 \mathrm{mg} / \mathrm{kg}$, p.o) was administered 2 hours following induction of colitis [25]. 
2.4. Induction of Colitis. Colonic inflammation was induced according to the method described by Morris et al. [26]. Animals were fasted for $36 \mathrm{~h}$ and slightly anesthetized with diethyl ether. Rats were thereafter positioned on their right side, and a single intracolonic dose of $10 \mathrm{mg}(50 \mathrm{mg} / \mathrm{kg})$ TNBS dissolved in $0.25 \mathrm{~mL}$ of $50 \%$ ethanol $(\mathrm{v} / \mathrm{v})$ was administered through a polyethylene catheter inserted $8 \mathrm{~cm}$ proximal to the anus. It was followed by maintaining rats in a head down position for 2-3 minutes to avoid immediate anal leakage of the instillate, and thereafter the rats were returned to their cages with access to food and water ad libitum. Normal groups were given an enema of $0.25 \mathrm{~mL}$ of normal saline.

2.5. Measurement of Body Weight Changes and Diarrheal Status. Animal body weights and occurrence of diarrhoea were recorded daily throughout all the experiments. Percent of body weight loss was thereafter measured. Using arbitrary criteria (1) formed stools, (2) loosed stools, (3) diarrhea, fecal output was assessed daily for 6 days.

2.6. Macroscopic Studies. Animals were sacrificed by means of ether inhalation on day 6 . For each animal, the distal colon was removed and cut longitudinally, slightly cleaned in physiological saline to remove fecal residues, weighed and processed for assessment by macroscopic, histological scores and biochemical markers. For each specimen, distal colon wet weight $(\mathrm{mg})(8 \mathrm{~cm}$ from the anus) and weight/length ratio $(\mathrm{mg} / \mathrm{cm}$ ) were measured. Using scoring system depicted in Table 1 according to the criteria of Ballester et al., with slight modifications, the severity of macroscopically visible colonic damage was scored [27]. Pieces of damaged colon thereafter were collected and immediately frozen in liquid nitrogen for measurement of biochemical parameters. After taking photos from distal colons, ulcer area and percent of necrosis were determined according to our previously described method [15].

2.7. Histological Studies. Cross-sections were selected and embedded in paraffin. Full-thickness sections of $4 \mu \mathrm{m}$ were obtained at different levels and stained with haematoxylin and eosin (H\&E). The histological damage was evaluated by a coworker pathologist, who was blinded to the study, according to the criteria previously described by Cooper et al. and Dieleman et al. $[28,29]$. Total colitis index was then derived by summing 3 subscores (inflammation severity, inflammation extent, and crypt damage) on H\&E-stained and coded sections.

2.8. MPO Activity Determination. Myeloperoxidase (MPO) activity was measured according to the technique described by Bradley et al. [30] with some modification. Each segment was weighed and chopped in $1 \mathrm{~mL}$ of $50 \mathrm{mM}$ potassium phosphate buffer involving 0.5\% HTAB. Having chopped, we placed tissue in a homogenizing tube. The container was then rinsed with $2 \times 1 \mathrm{~mL}$ HTAB in buffer solution. Afterwards, we added more buffer in order to have a concentration which was equivalent to $5 \mathrm{~mL}$ per $0.1 \mathrm{~g}$ of
TABLE 1: Scoring criteria for assessment of macroscopic rat colonic injuries.

\begin{tabular}{ll}
\hline & 0 No adhesions \\
& 1 Difficult dissection \\
& 2 Visible adhesions \\
& 3 "Wrapped" intestine \\
& 0 No obstruction \\
& 1 Need for gentle manual cleaning \\
& 2 Fecal impaction \\
\hline Obstruction & 0 Similar to uninflamed intestine \\
& 1 Thicker than normal $(\sim 1-2 \mathrm{~mm})$ \\
& 2 Much thicker than normal $(>2 \mathrm{~mm})$ \\
\hline Thickening & 0 Similar to uninflamed intestine \\
& 1 Mild and generalized or intense but \\
& localized hyperemia \\
& 2 Intense and localized hyperemia \\
& 3 Frank hemorrhage \\
\hline Hyperemia & 0 No signs of necrosis \\
& 1 Small areas of necrosis \\
& 2 Patchy necrosis \\
& 3 Focal necrosis $<0.8 \mathrm{~cm}$ \\
& 4 Focal necrosis $>0.8 \mathrm{~cm}$ \\
& 5 Extended necrotic lesion \\
\hline Necrosis &
\end{tabular}

colon tissue and homogenized (15,000 rpm) for $4 \times 45 \mathrm{~s}$ at $1 \mathrm{~min}$ intervals. The homogenate was placed in a sample tube, sonicated in an ice bath for $10 \mathrm{~s}$, subjected to 3 cycles of freezing and thawing, and sonicated again for $10 \mathrm{~s}$. The suspensions were centrifuged $(15,000 \mathrm{rpm}$ for $15 \mathrm{~min}$ in $4^{\circ} \mathrm{C}$ ). The supernatant thereafter decanted for assessment. The MPO activity was analyzed spectrophotometrically as follows: $0.1 \mathrm{~mL}$ of the supernatant was added to $2.9 \mathrm{~mL}$ of $50 \mathrm{mM} \mathrm{K}_{3} \mathrm{PO}_{4}$ buffer $(\mathrm{pH}=6.0)$ involving O-dianisidinedihydrochloride $(0.167 \mathrm{mg} / \mathrm{mL})$ and $0.005 \%$ hydrogen peroxide. The absorbance of the reaction mixture was recorded at a wave length of $450 \mathrm{~nm}$ by means of a UV-Vis spectrophotometer. The results are expressed as the change in absorbance $/ \mathrm{min} / \mathrm{mg}$ colonic wet weight.

2.9. Cytokine Assays. The amount of Rat TNF- $\alpha$, IL- $1 \beta$, and IL- 6 in the colonic samples was quantified by commercially available enzyme-linked immunosorbent assay kits (ALPCO, USA) as described earlier [31].

\section{Statistical Analysis}

All data are expressed as mean \pm S.E.M. Clinical activity score of colitis and macroscopic and histological scores were statistically analyzed using the Mann-Whitney $U$ test. Differences in parametric data were determined by one-way analysis of variance (ANOVA) with TUKEY as post hoc test. Differences were considered statistically significant with $P<0.05$. 
TABLE 2: Effect of cisapride (2 mg/kg, i.p; $4 \mathrm{mg} / \mathrm{kg}$, p.o: daily) on macroscopic and histopathological parameters of the rat colon 6 days after induction of colitis with TNBS $(50 \mathrm{mg} / \mathrm{kg})$.

\begin{tabular}{|c|c|c|c|c|c|c|}
\hline Group & $\begin{array}{l}\text { Colonic weight/length ratio } \\
(\mathrm{mg} / \mathrm{cm})\end{array}$ & $\begin{array}{c}\text { Body weight loss after } \\
6 \text { days }(\%)\end{array}$ & $\begin{array}{c}\text { Ulcer severity } \\
(0-15)\end{array}$ & $\begin{array}{l}\text { Total colitis index } \\
(0-10) \\
\end{array}$ & $\begin{array}{c}\text { Ulcer area } \\
\left(\mathrm{cm}^{2}\right)\end{array}$ & $\begin{array}{c}\text { Necrosis } \\
(\%)\end{array}$ \\
\hline Normal (i.p) & $64.8 \pm 2.3$ & $-1.9 \pm 0.6$ & 0.0 & 0.0 & 0.0 & 0.0 \\
\hline Normal (p.o) & $72.1 \pm 2.8$ & $-1.6 \pm 0.5$ & 0.0 & 0.0 & 0.0 & 0.0 \\
\hline TNBS control (i.p) & $256.3 \pm 9.5$ & $7.7 \pm 0.7$ & $12.2 \pm 0.6$ & $9.9 \pm 0.1$ & $6.2 \pm 0.1$ & $51.5 \pm 2.8$ \\
\hline TNBS control (p.o) & $252.1 \pm 9.4$ & $8.2 \pm 0.8$ & $11.8 \pm 0.7$ & $9.9 \pm 0.1$ & $6.0 \pm 0.2$ & $48.8 \pm 3.8$ \\
\hline Cis. $(2 \mathrm{mg} / \mathrm{kg}$, i.p $)$ & $256.3 \pm 7.1$ & $7.1 \pm 0.6$ & $12.0 \pm 0.5$ & $9.8 \pm 0.1$ & $5.7 \pm 0.3$ & $48.2 \pm 3.5$ \\
\hline Cis (4 mg/kg, p.o) & $253.1 \pm 8.2$ & $8.0 \pm 0.9$ & $12.2 \pm 0.5$ & $9.5 \pm 0.2$ & $6.0 \pm 0.3$ & $50.0 \pm 1.8$ \\
\hline Dex. (1 mg/kg, i.p) & $161.9 \pm 27.4^{* *}$ & $3.9 \pm 0.3^{*}$ & $5.33 \pm 1.4^{* *}$ & $4.8 \pm 0.8^{* *}$ & $4.0 \pm 0.7^{* *}$ & $27.0 \pm 7.9^{* *}$ \\
\hline Dex. $(2$ mg/kg, p.o $)$ & $163.5 \pm 22.9^{\# \#}$ & $4.7 \pm 0.8^{\#}$ & $4.7 \pm 1.4^{\# \#}$ & $4.9 \pm 0.5^{\# \#}$ & $3.9 \pm 0.3^{\# \#}$ & $22.2 \pm 5.2^{\# \#}$ \\
\hline
\end{tabular}

TNBS, 2, 4, 6-trinitrobenzenesulfonic acid, cis: cisapride, dex.: dexamethasone, i.p: intraperitoneal, p.o: oral.

Values are means \pm SEM: $n=6$.

${ }^{*} P<0.01$ and ${ }^{*} P<0.05$ : significant difference compared to TNBS-control group, i.p; ${ }^{\#} P<0.01$ and ${ }^{\#} P<0.05$ : significant difference compared to TNBScontrol group, p.o.

\section{Results}

4.1. Changes in Rats' Body Weight and Diarrheal Status. TNBS- and cisapride-treated rats (i.p, p.o) showed loss of body weight after 6 days $(P<0.001)$, and there was no significant difference between these groups in aforementioned parameter. Animals in dexamethasone-treated groups (i.p, p.o) also experienced a significant loss of body weight in comparison with normal group; however, percent of body weight loss in these groups was significantly lower than TNBS-control or cisapride groups after 6 days $(P<0.05)$ (Table 2). Furthermore, there were no significant differences between i.p and p.o of either medication.

As can be noted in Figure 1, diarrheal status in TNBSand cisapride-treated rats during all 6 days of the experiment was significantly high, in comparison to normal groups $(P<$ 0.01). No significant difference was observed in the daily diarrheal status between animals treated with cisapride and TNBS over the experiment.

Additionally, rats treated with dexamethasone exhibited a significant decrease in the diarrhea index especially after the initial 2 days of treatment subsequent to induction of colitis, in comparison with TNBS-control group (at least $P<0.05$ ).

4.2. Effect of Cisapride on Macroscopic Features. TNBScontrol and cisapride groups (i.p, p.o) experienced severe inflammation, hemorrhage, ulcer, necrosis, and thickened colon wall 6 days after induction of colitis, while the normal macroscopic features were evident in colons of normal group (Table 2, Figure 2). There was also no significant difference in these variables between TNBS and cisapride-treated rats. Compared with TNBS-control group, there was a significant decrease in ulcer severity, weight/length ratio, ulcer area, and percent of necrosis in dexamethasone-(i.p, p.o) treated groups $(P<0.01)$. There were no significant differences between i.p and p.o of either medication.

4.3. Effect of Cisapride on Histopathological Features. Figure 3 depicts histological assessment in studied groups, 6 days subsequent to induction of colitis. Normal group showed

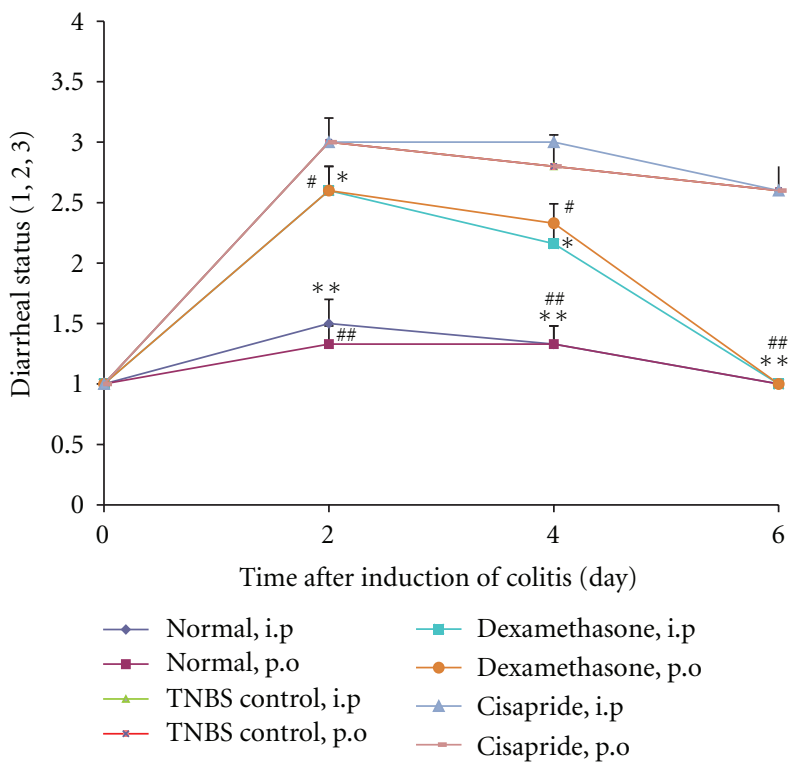

Figure 1: Changes in diarrheal status before (day 0 ) and during 6 days of treatment after induction of colitis (TNBS, $50 \mathrm{mg} / \mathrm{kg}$ ) in rats. Values are means $\pm \operatorname{SEM}(n=6)$. ${ }^{* *} P<0.01$ and ${ }^{*} P<0.05$ compared with TNBS-control group, i.p; ${ }^{\# \#} P<0.01$ and ${ }^{\#} P<0.05$ compared with TNBS-control group, p.o TNBS, 2, 4, 6trinitrobenzenesulfonic acid. Stool consistency was daily checked in rats (1) formed stools, (2) loosed stools, and (3) diarrhea.

a normal architecture with intact epithelium in colonic mucosa. There were severe and intense transmural inflammation and/or diffuse necrosis, inflammatory granulomas, and submucosal neutrophils infiltration in TNBS-control $(50 \mathrm{mg} / \mathrm{kg}$ ) and cisapride (i.p, p.o) group. There was no significant difference in microscopic features between cisapridetreated and TNBS-control groups.

Dexamethasone-treated groups (i.p, p.o) significantly showed less histopathological damages. These treatments reduced total colitis index (inflammation severity, inflammation extent, and crypt damage) in injurious colons (Table 2). 


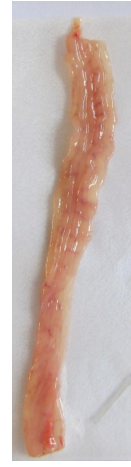

(a)

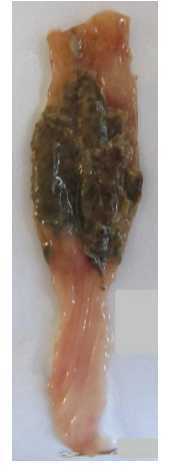

(b)

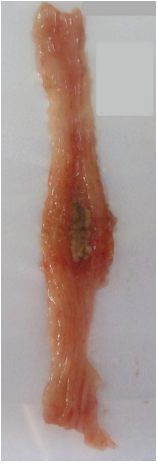

(c)

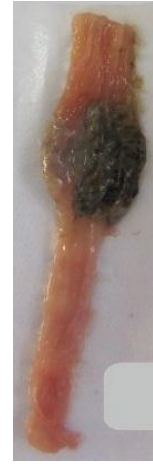

(d)

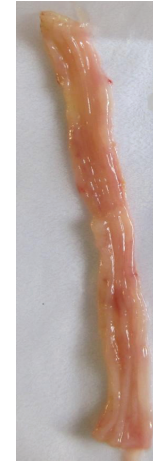

(e)

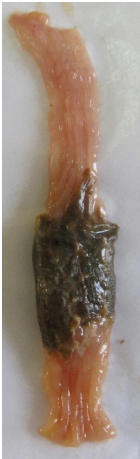

(f)

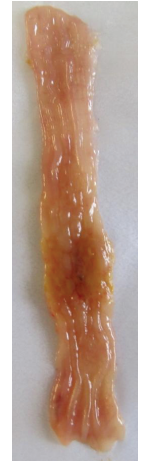

(g)

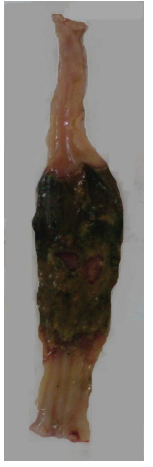

(h)

Figure 2: Macroscopic presentation of TNBS-induced colitis in rats. (a) normal rat, i.p; (b)TNBS-control rat, i.p (50 mg/kg); (c) dexamethasone-treated rat, i.p (1 mg/kg); (d) cisapride-treated rat, i.p (2 mg/kg); (e) normal rat, p.o; (f) TNBS-control rat, p.o (50 mg/kg); (g) dexamethasone-treated rat, p.o $(2 \mathrm{mg} / \mathrm{kg})$; (h) cisapride-treated rat, p.o (4 mg/kg). Note the extensive hyperemia, edema, ulceration, visible enlargement, and necrosis of the colon in B, D, F, and $\mathrm{H}$ groups. In C and G groups the damage score of colon was significantly decreased.

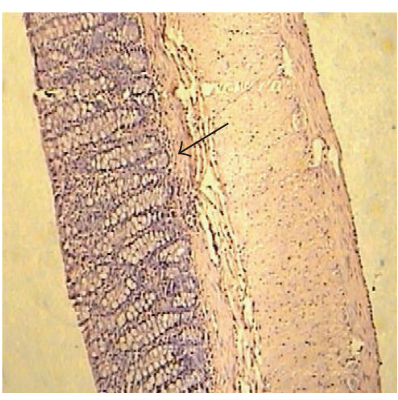

(a)

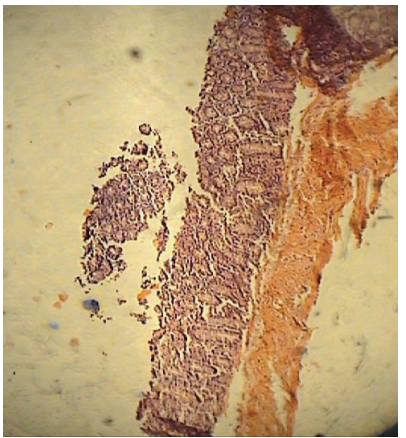

(e)

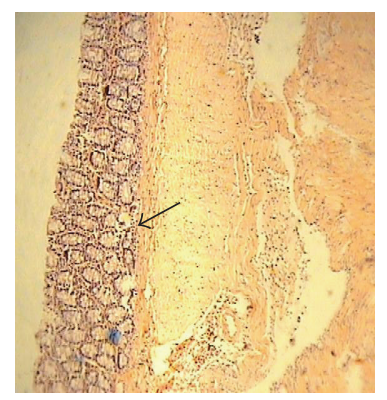

(b)

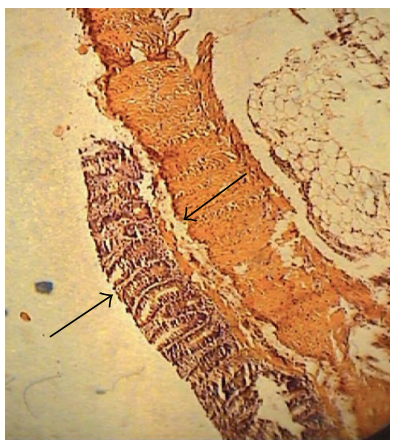

(f)

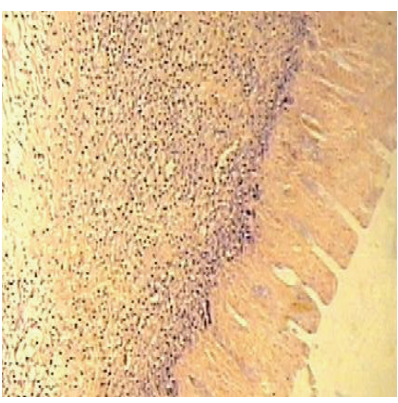

(c)

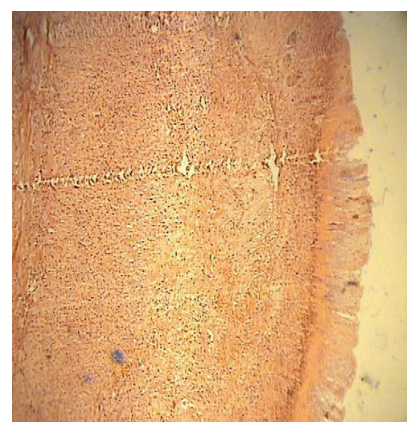

(g)

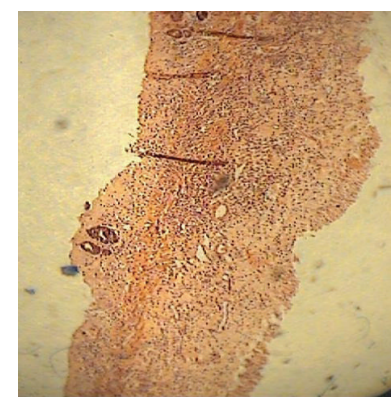

(d)

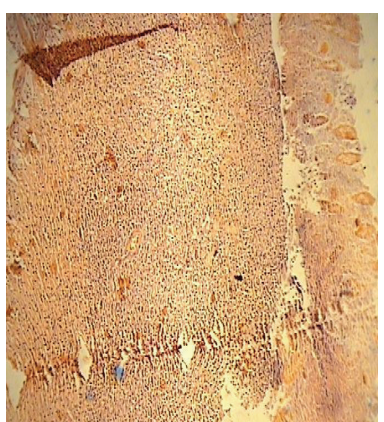

(h)

Figure 3: Microscopic presentation of TNBS-induced colitis in rats (hematoxylin and eosin staining; original magnification $\times 10)$. $(\mathrm{a}$ and $\mathrm{b}$ ) Normal groups: mucus layer and crypts are normal; (c and d) TNBS-control groups: epithelial distortion, crypt damage, and inflammatory cell infiltrates; (e and f) dexamethasone (i.p and p.o, resp.): moderate mucosal and submucosal inflammation and mucosal inflammatory cell infiltrates; (g and h): cisapride (i.p and p.o, resp.) infiltration of neutrophils and destruction of mucosal architecture.

Furthermore, these groups experienced reepithelization of the mucosal layer and reduced inflammatory cell infiltration in lamina propria. There were no significant differences between i.p and p.o of either medication.

4.4. Effect of Cisapride on Myeloperoxidase Activity. As can be shown in Table 3, MPO activity, a marker for leukocyte infiltration into the inflamed tissue, was markedly enhanced in the inflamed colons subsequent to the intrarectal TNBS administration versus normal groups. This result confirmed, the histological assessment which showed increased leucocyte infiltration in TNBS-control groups. Administration of dexamethasone (i.p, p.o) significantly declined the MPO activity level $(P<0.01)$. There were no significant differences 
TABLE 3: Biochemical parameters of the rat colon 6 days after induction of colitis with TNBS (50 mg/kg).

\begin{tabular}{lcccc}
\hline Group & $\begin{array}{c}\text { MPO activity } \\
\text { (unit/100 mg wet tissue) }\end{array}$ & $\begin{array}{c}\text { TNF- } \alpha \\
\text { (pg/g wet tissue) }\end{array}$ & $\begin{array}{c}\text { IL-6 } \\
\text { (pg/g wet tissue) }\end{array}$ & $\begin{array}{c}\text { IL-1 } \beta \\
\text { (pg/g wet tissue) }\end{array}$ \\
\hline Normal (i.p) & $0.6 \pm 0.1$ & $141.0 \pm 13.8$ & $3663.3 \pm 240.2$ & $1647.5 \pm 452.4$ \\
Normal (p.o) & $0.6 \pm 0.1$ & $144.6 \pm 12.7$ & $3646.7 \pm 261.0$ & $1768.7 \pm 578.8$ \\
TNBS control (i.p) & $3.4 \pm 0.4$ & $249.9 \pm 26.6$ & $5210.3 \pm 457.9$ & $15296.0 \pm 1579.7$ \\
TNBS control (p.o) & $3.4 \pm 0.4$ & $251.8 \pm 18.5$ & $5272.0 \pm 364.5$ & $15563.4 \pm 1476.5$ \\
Cis (2 mg/kg, i.p) & $3.5 \pm 0.3$ & $254.1 \pm 21.5$ & $5133.7 \pm 352.2$ & $15378.3 \pm 1252.5$ \\
Cis (4 mg/kg, p.o) & $3.6 \pm 0.2$ & $251.6 \pm 15.6$ & $5224.8 \pm 263.9$ & $15825.3 \pm 1198.2$ \\
Dex. (1 mg/kg, i.p) & $1.9 \pm 0.4^{* *}$ & $160.6 \pm 12.6^{*}$ & $3821.7 \pm 270.7^{*}$ & $8641.2 \pm 985.2^{* *}$ \\
Dex. (2 mg/kg, p.o) & $1.8 \pm 0.1^{\# \#}$ & $164.7 \pm 10.1^{\#}$ & $3870.7 \pm 240.2^{\#}$ & $9162.7 \pm 1574.4^{\#}$ \\
\hline
\end{tabular}

TNBS: 2, 4, 6-trinitrobenzenesulfonic acid, cis: cisapride, dex.: dexamethasone, i.p: intraperitoneal, p.o: oral.

Values are means \pm SEM: $n=6$.

${ }^{* *} P<0.01$ and ${ }^{*} P<0.05$ : significant difference compared to TNBS-control group, i.p; ${ }^{\# \#} P<0.01$ and ${ }^{\#} P<0.05$ : significant difference compared to TNBScontrol group, p.o.

between i.p and p.o of either medication or no differences between cisapride and TNBS administered with the same route.

4.5. Effect of Cisapride on Cytokines Profile. As can be noted in Table 3, TNF- $\alpha$, IL-1 $\beta$, and IL- 6 contents soared in the TNBS-control groups, as compared with those of normal rats. Cisapride-treated groups (i.p, p.o) did not experience any significant changes in the levels of inflammatory cytokines compared with TNBS-control groups. These parameters were significantly lowered in rats treated with dexamethasone (i.p, p.o) (Table 3). In addition, there were no significant differences between i.p and p.o of either medication.

\section{Discussion}

The present study assessed the effects of administration of cisapride on the severity of experimental colitis in rats. Cisapride-treated rats showed a colitis which was comparable to that of TNBS-control group. In fact, evaluation of clinical, macroscopic, histopathologic, and biochemical parameters showed that there was no significant difference between cisapride and TNBS-control groups.

A suitable animal model of IBD is to show all the characteristics of a typical human IBD such as macroscopic, histopathological, and biochemical alternations accompanied by covering acute and chronic symptoms. Of the different models of induction of colitis, TNBS-induced colitis is one of the best which can mimic efficiently the pattern of inflammation similar to human ulcerative colitis. This model creates a simple process, reproducible colonic damage, and long-lasting damage accompanied by inflammatory cell infiltration and ulcers. Taking into account the fact that serotonin increase has been demonstrated in TNBS-induced colitis, this model is likely to be beneficial and suitable for analyzing the drugs which impact serotonin pathway in intestinal inflammation $[15,16,32]$. An increase was found in the bioavailability of 5-HT from mucosal epithelial cells in TNBS-induced colitis [16]. In addition, TNBS model could activate both $T h_{1}$ and $T h_{2}$ responses [33]. It can also induce both acute and chronic phases of colitis predicated upon experiment period [34]. Therefore, present study set out as a six-day experiment to assess the influence of chronic administration of cisapride on TNBS-induced colitis. The time period adopted in the present experiment was similar to the previous studies carried out on model of TNBS-induced chronic inflammation in rats [26].

An increase in the number of EC cells and 5-HT content was shown to be involved in intestinal mucosal inflammation such as ulcerative colitis [18]. It should be mentioned that serotoninergic receptors were found in immune cells including macrophages which are principle source of proinflammatory cytokines IL-1, IL-6, and TNF [19]. On the grounds of strategic location of EC, it can be inferred that 5-HT plays a part in infiltration and activation of macrophages in intestinal inflammation. Serotonin is also secreted by immune cells; hence, this exogenously added serotonin increases T-cell proliferation [19]. T cells are obviously involved in pathogenesis of IBD. Not only does $\mathrm{Th}_{1}$ cell activation liberate proinflammatory cytokines, but also it stimulates tissue macrophages in order to release additional proinflammatory cytokines (e.g., TNF- $\alpha$, IL- $1 \beta$, IL-6, IL-8, and IL-12), nitric oxide, and reactive oxygen species [35]. Amongst the intestinal immunomodulatory factors, cytokines are believed to serve a crucial role. To clarify, an imbalance between proinflammatory and antiinflammatory cytokines is associated with the IBD pathogenesis. Proinflammatory cytokines (e.g., TNF- $\alpha$, IL- $1 \beta$, and IL6) liberated from macrophages, neutrophils, and endothelial cells were shown to be overproduced in TNBS-induced colitis and in human IBD [36]. It should be noted that they can liberate other cytokines, arachidonic acid metabolites, and lytic enzymes by intestinal macrophages, neutrophils, smooth muscle cells, fibroblasts, and epithelial cells and thus result in edema, fibrosis, and necrosis [37]. Furthermore, TNF- $\alpha$ and IL- $1 \beta$ are the main mediators pertaining to neutrophil activation and mobilization [37]. In the present study, we found that the level of these proinflammatory cytokines increased subsequent to TNBS instillation. In addition, our findings reported that the colonic levels of these 
mediators are still high in cisapride-treated rats. This result is possibly due to stimulation of synthesis and/or release of these mediators.

MPO activity has been used as a quantitative index of neutrophil influx into inflamed intestinal tissue [36]. In the preset study, MPO activity was conspicuously enhanced in TNBS-treated rats, and there was no significant difference in MPO activity between TNBS-control and cisapride groups. This high level of MPO activity and infiltration of inflammatory cells into the colonic tissue is consistent with severe macroscopic and microscopic damage scores in these groups.

Most 5-HT is released from EC cells, and therefore the majority accumulation of 5HT is in GI tract. In addition, it has been demonstrated that an increase in the number of EC cells and in 5-HT content is associated with intestinal mucosal inflammation such as ulcerative colitis [18]. It is also worth nothing that amongst the variety of serotoninergic receptors, $5 \mathrm{HT}_{4}$ receptors exert extensive distribution in GI. In fact the highest expression of $5 \mathrm{HT}_{4}$ receptors is in the distal colon [17].

Based on the probable association between 5HT and intestinal inflammation and also the extensive distribution of $5 \mathrm{HT}_{4}$ receptors in colon, it can be inferred that this class of receptor maybe has a fundamental role in the pathogenesis of UC. It would be expected following the administration of cisapride as a $5 \mathrm{HT}_{4}$ receptor agonist, liberation of $5 \mathrm{HT}$, and activation of $5 \mathrm{HT}$ receptors on macrophages that the severity of experimental TNBS-induced colitis would be aggravated. Our results showed that the severity of colitis in TNBScontrol group, and cisapride-treated rats was similar and cisapride did not deteriorate the severity of experimental colitis in rat. This is likely to arise from the severity of colitis that reached its peak about 6 days following colitis induction, and no more severity can stem from cisapride which is a potent $5 \mathrm{HT}_{4}$ receptor agonist. On the other hand, we must notice that cisapride dosage was selected on the basis of previous studies using this drug to analyze the role of 5- $\mathrm{HT}_{4}$ receptors in GI function in rat, and amongst these employed doses we selected the high dose of cisapride in our study. Therefore, it seems that dose of cisapride would not be a probable limitation in this work. Furthermore, it can be deduced that because the severity of colitis produced by TNBS is massive (through various pathways), it is likely that cisapride could not bring about more colitis damages through $5 \mathrm{HT}_{4}$ receptors. In fact for determination of the role of $5 \mathrm{HT}_{4}$ receptor in the pathogenesis of colitis, alongside the assessment of the effect of $5 \mathrm{HT}_{4}$ receptor activation, it should be better to investigate the effect of blocking of this receptor on the severity of experimental colitis. Therefore we suggest further researches using administration of one $5 \mathrm{HT}_{4}$ antagonist and evaluation of its effects on severity of experimental colitis. If the administration of a $5 \mathrm{HT}_{4}$ receptor antagonist could ameliorate the colonic injuries and also cisapride could antagonize the probable beneficial effect of $5 \mathrm{HT}_{4}$ receptor antagonist, the important role of $5 \mathrm{HT}_{4}$ receptors in the pathogenesis of colitis will be revealed.

In conclusion, administration of cisapride, a $5 \mathrm{HT}_{4}$ receptor agonist, does not aggravate the severity of colonic injuries in TNBS-induced colitis in rat. Further studies are required using administration of $5 \mathrm{HT}_{4}$ receptor agonist/antagonist to investigate the exact role of $5 \mathrm{HT}_{4}$ receptors in the pathogenesis of ulcerative colitis.

\section{Acknowledgment}

This work was financially supported by a grant project no. 389362 by Vice Chancellor for Research, Isfahan University of Medical Sciences.

\section{References}

[1] B. E. Sands, "Therapy of inflammatory bowel disease," Gastroenterology, vol. 118, no. 2, pp. S68-S82, 2000.

[2] D. K. Podolsky, "Inflammatory bowel disease," The New England Journal of Medicine, vol. 347, pp. 417-429, 2002.

[3] D. C. Baumgart and W. J. Sandborn, "Inflammatory bowel disease: clinical aspects and established and evolving therapies," Lancet, vol. 369, no. 9573, pp. 1641-1657, 2007.

[4] E. Forbes, T. Murase, M. Yang et al., "Immunopathogenesis of experimental ulcerative colitis is mediated by eosinophil peroxidase," Journal of Immunology, vol. 172, no. 9, pp. 56645675, 2004.

[5] M. B. Grisham, "Molecular and cellular aspects of intestinal inflammation: clinical implications in inflammatory bowel disease," Inflammatory Bowel Diseases, vol. 1, pp. 1-14, 2000.

[6] L. Langmead and D. S. Rampton, "Review article: complementary and alternative therapies for inflammatory bowel disease," Alimentary Pharmacology and Therapeutics, vol. 23, no. 3, pp. 341-349, 2006.

[7] I. Cloëz-Tayarani, A. F. Petit-Bertron, H. D. Venters, and J. M. Cavaillon, "Differential effect of serotonin on cytokine production in lipopolysaccharide-stimulated human peripheral blood mononuclear cells: involvement of 5-hydroxytryptamine2A receptors," International Immunology, vol. 15, no. 2, pp. 233-240, 2003.

[8] D. Y. Kim and M. Camilleri, "Serotonin: a mediator of the brain-gut connection," American Journal of Gastroenterology, vol. 95, no. 10, pp. 2704-2709, 2000.

[9] M. D. Gershon and J. Tack, “The serotonin signaling system: from basic understanding to drug development for functional GI disorders," Gastroenterology, vol. 132, no. 1, pp. 397-414, 2007.

[10] M. M. Costedio, N. Hyman, and G. M. Mawe, "Serotonin and its role in colonic function and in gastrointestinal disorders," Diseases of the Colon and Rectum, vol. 50, no. 3, pp. 376-388, 2007.

[11] M. G. Kris, "Ondansetron: a specific serotonin antagonist for the prevention of chemotherapy-induced vomiting," Important Advances in Oncology, pp. 165-177, 1994.

[12] F. De Ponti, "Pharmacology of serotonin: what a clinician should know," Gut, vol. 53, no. 10, pp. 1520-1535, 2004.

[13] G. J. McCleane, R. Suzuki, and A. H. Dickenson, "Does a single intravenous injection of the $5 \mathrm{HT} 3$ receptor antagonist ondansetron have an analgesic effect in neuropathic pain? A double-blinded, placebo-controlled cross-over study," Anesthesia and Analgesia, vol. 97, no. 5, pp. 1474-1478, 2003. 
[14] P. Hrycaj, "Serotonin type 3 receptor antagonist tropisetron in the treatment of chronic inflammatory rheumatic conditions-preliminary clinical experience," Scandinavian Journal of Rheumatology, vol. 33, no. 119, pp. 55-58, 2004.

[15] A. Motavallian-Naeini, S. Andalib, M. Rabbani, P. Mahzouni, M. Afsharipour, and M. Minaiyan, "Validation and optimization of experimental colitis induction in rats using 2,4, 6-trinitrobenzene sulfonic acid," Research in Pharmaceutical Sciences, vol. 7, no. 3, pp. 159-169, 2012.

[16] D. R. Linden, J. X. Chen, M. D. Gershon, K. A. Sharkey, and G. M. Mawe, "Serotonin availability is increased in mucosa of guinea pigs with TNBS-induced colitis," American Journal of Physiology, vol. 285, no. 1, pp. G207-G216, 2003.

[17] J. M. Hoffman, K. Tyler, S. J. MacEachern et al., "Activation of colonic mucosal 5- $\mathrm{HT}_{4}$ receptors accelerates propulsive motility and inhibits visceral hypersensitivity," Gastroenterology, vol. 142, no. 4, pp. 844-854, 2012.

[18] M. D. Coates, C. R. Mahoney, D. R. Linden et al., "Molecular defects in mucosal serotonin content and decreased serotonin reuptake transporter in ulcerative colitis and irritable bowel syndrome," Gastroenterology, vol. 126, no. 7, pp. 1657-1664, 2004.

[19] B. L. Fiebich, R. S. Akundi, M. Seidel et al., "Expression of 5HT3A receptors in cells of the immune system," Scandinavian Journal of Rheumatology, vol. 33, no. 119, pp. 9-11, 2004.

[20] J. Stefulj, B. Jernej, L. Cicin-Sain, I. Rinner, and K. Schauenstein, "mRNA expression of serotonin receptors in cells of the immune tissues of the rat," Brain, Behavior, and Immunity, vol. 14, no. 3, pp. 219-224, 2000.

[21] J. E. Ghia, N. Li, H. Wang et al., "Serotonin has a key role in pathogenesis of experimental colitis," Gastroenterology, vol. 137, no. 5, pp. 1649-1660, 2009.

[22] A. Motavallian-Naeini, M. Minaiyan, M. Rabbani, and P. Mahzuni, "Anti-inflammatory effect of ondansetron through 5 HT3 receptors on TNBS-induced coloitis in rat," Experimental and Clinical Sciences Journal, vol. 11, pp. 30-44, 2012.

[23] M. Minaiyan, A. R. Ghannadi, M. Etemad, and P. Mahzouni, "A study of the effects of Cydonia oblonga Miller (Quince) on TNBS-induced ulcerative colitis in rats," Research in Pharmaceutical Sciences, vol. 7, no. 2, pp. 103-110, 2012.

[24] A. Tari, K. Sumii, M. Yoshihara et al., "Effect of cisapride on the concentrations of $\beta$-endorphinlike immunoreactivity and substance P-like immunoreactivity in the rat gastrointestinal tract," Biochemical and Biophysical Research Communications, vol. 147, no. 3, pp. 1162-1169, 1987.

[25] E. García-Garayoa, A. Monge, J. Roca, J. Del Río, and B. Lasheras, "VB20B7, a novel 5-HT-ergic agent with gastrokinetic activity-II. Evaluation of the gastroprokinetic activity in rats and dogs," Journal of Pharmacy and Pharmacology, vol. 49, no. 1, pp. 66-73, 1997.

[26] G. P. Morris, P. L. Beck, M. S. Herridge, W. T. Depew, M. R. Szewczuk, and J. L. Wallace, "Hapten-induced model of chronic inflammation and ulceration in the rat colon," Gastroenterology, vol. 96, no. 3, pp. 795-803, 1989.

[27] I. Ballester, A. Daddaoua, R. López-Posadas et al., “The bisphosphonate alendronate improves the damage associated with trinitrobenzenesulfonic acid-induced colitis in rats," British Journal of Pharmacology, vol. 151, no. 2, pp. 206-215, 2007.

[28] H. S. Cooper, S. N. S. Murthy, R. S. Shah, and D. J. Sedergran, "Clinicopathologic study of dextran sulfate sodium experimental murine colitis," Laboratory Investigation, vol. 69, no. 2, pp. 238-250, 1993.
[29] L. A. Dieleman, M. J. H. J. Palmen, H. Akol et al., "Chronic experimental colitis induced by dextran sulphate sodium (DSS) is characterized by Th1 and Th2 cytokines," Clinical and Experimental Immunology, vol. 114, no. 3, pp. 385-391, 1998.

[30] P. P. Bradley, D. A. Priebat, R. D. Christensen, and G. Rothstein, "Measurement of cutaneous inflammation: estimation of neutrophil content with an enzyme marker," Journal of Investigative Dermatology, vol. 78, no. 3, pp. 206-209, 1982.

[31] V. Pereira Nacife, M. de Nazaré Correia Soeiro, R. Novaes Gomes, H. D'Avila, H. Caire Castro-Faria Neto, and M. de Nazareth Leal Meirelles, "Morphological and biochemical characterization of macrophages activated by carrageenan and lipopolysaccharide in vivo," Cell Structure and Function, vol. 29, no. 2, pp. 27-34, 2004.

[32] L. Zheng, Z. Q. Gao, and S. X. Wang, "A chronic ulcerative colitis model in rats," World Journal of Gastroenterology, vol. 6, no. 1, pp. 150-152, 2000.

[33] T. Dohi and K. Fujihashi, "Type 1 and 2 T helper cell-mediated colitis," Current Opinion in Gastroenterology, vol. 22, no. 6, pp. 651-657, 2006.

[34] M. N. Ajuebor, C. M. Hogaboam, S. L. Kunkel, A. E. I. Proudfoot, and J. L. Wallace, "The chemokine RANTES is a crucial mediator of the progression from acute to chronic colitis in the rat," Journal of Immunology, vol. 166, no. 1, pp. 552-558, 2001.

[35] I. Cloëz-Tayarani and J. P. Changeux, "Nicotine and serotonin in immune regulation and inflammatory processes: a perspective," Journal of Leukocyte Biology, vol. 81, no. 3, pp. 599-606, 2007.

[36] H. Esmaily, A. Hosseini-Tabatabaei, R. Rahimian et al., "On the benefits of silymarin in murine colitis by improving balance of destructive cytokines and reduction of toxic stress in the bowel cells," Central European Journal of Biology, vol. 4, no. 2, pp. 204-213, 2009.

[37] R. Maunder, "Mediators of stress effects in inflammatory bowel disease: not the usual suspects," Journal of Psychosomatic Research, vol. 48, no. 6, pp. 569-577, 2000. 


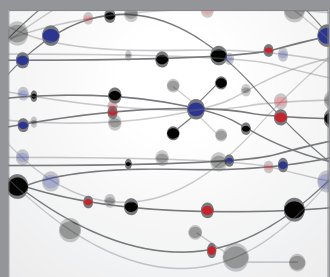

The Scientific World Journal
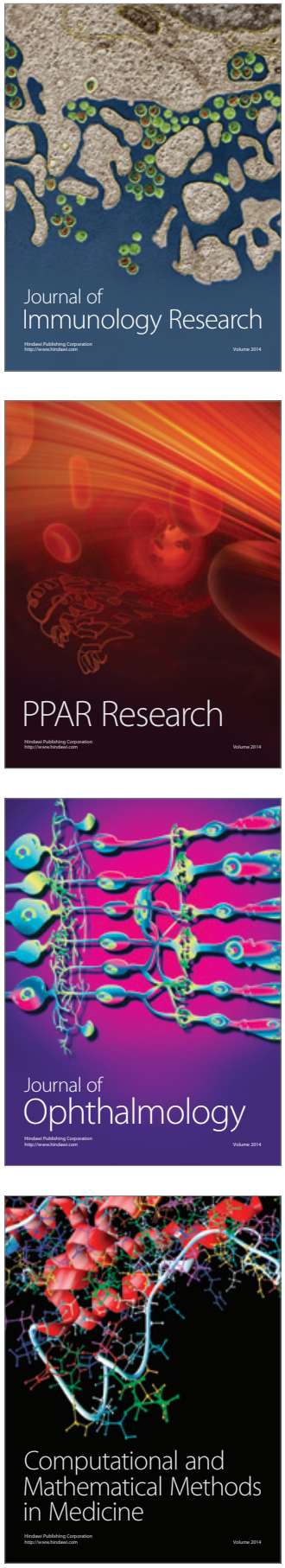

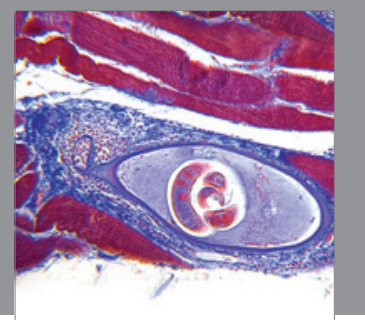

Gastroenterology

Research and Practice
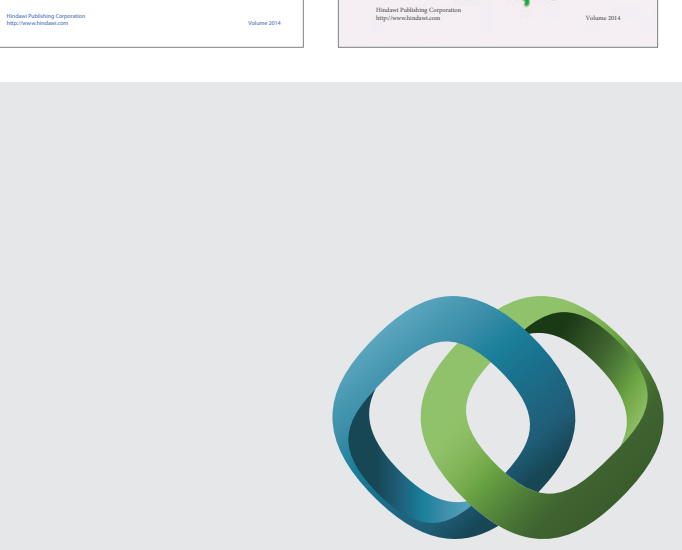

\section{Hindawi}

Submit your manuscripts at

http://www.hindawi.com
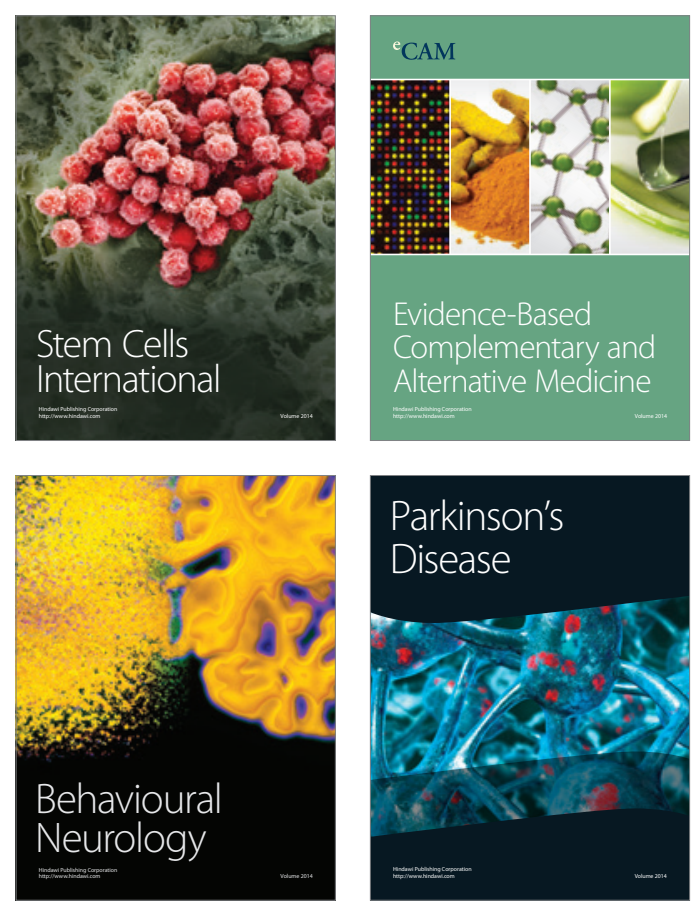

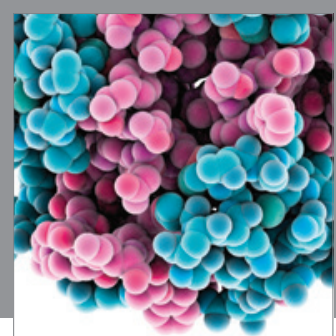

Journal of
Diabetes Research

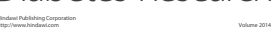

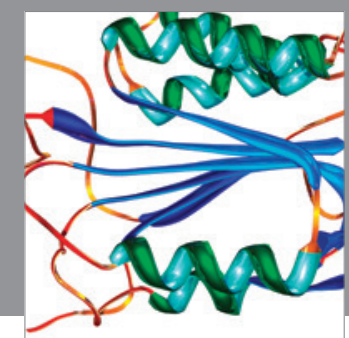

Disease Markers
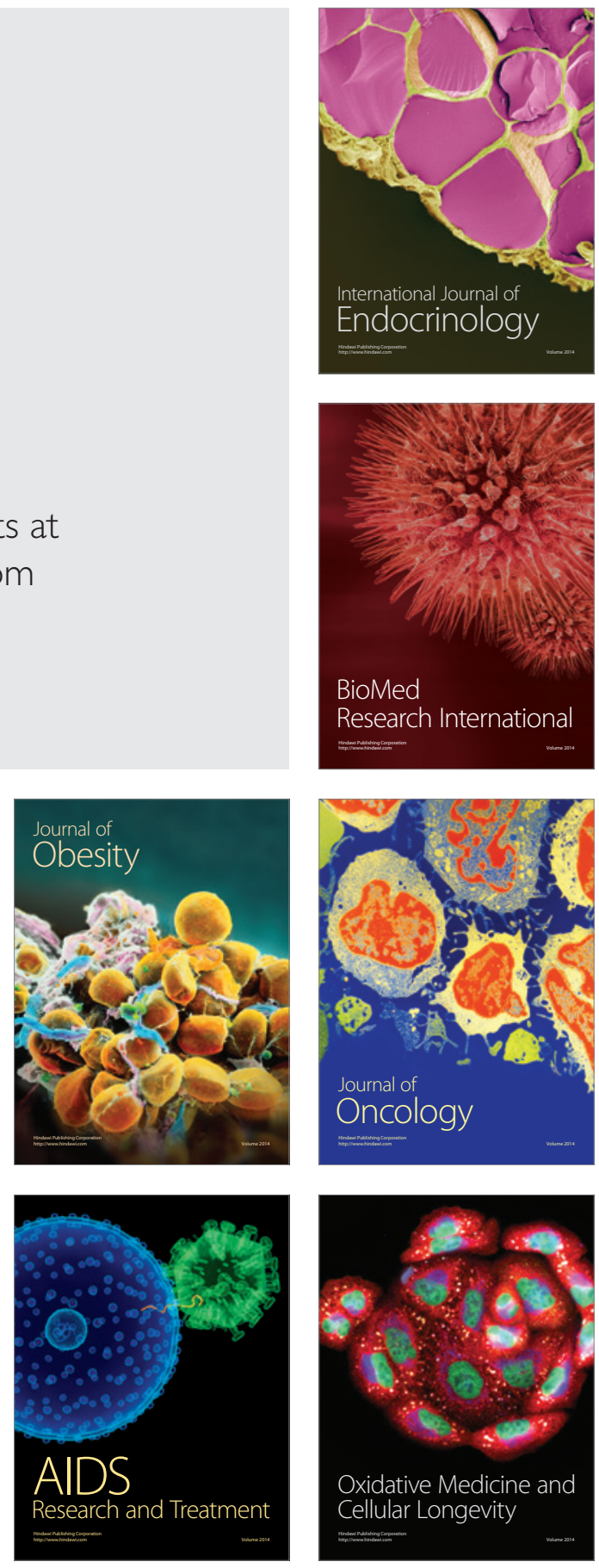